\title{
The sintering and densification behaviour of many copper nanoparticles: a molecular dynamics study
}

\author{
Bingqing Cheng ${ }^{\S}$ and Alfonso H.W. Ngan \\ Department of Mechanical Engineering, The University of Hong Kong, Pokfulam Road, \\ Hong Kong, P.R. China \\ ${ }^{\S}$ Correspondence author (email: tonicbq@gmail.com)
}

\begin{abstract}
Despite its relevance to a range of technological applications including nanocrystalline material fabrication, the sintering mechanisms of nanoparticles have not been well understood. It has been recognized that extrapolation from understanding of macro-particle sintering is unreliable for the nano-particle size regime. In this work, the sintering behaviour of copper nanoparticles under periodic boundary conditions at different temperatures and pressures was investigated by Molecular Dynamics simulations. It was found that smaller particle sizes, higher temperature and higher external pressure facilitate densification. Through a comparison with a two-sphere model, the governing mechanisms for many nanoparticles sintered at low temperature $(T \leq 900 K)$ were identified to be a variety of plasticity processes including dislocation, twinning and even amorphization at the contact neck regions, due to the presence of high stresses.
\end{abstract}

\section{Keywords:}

Nanoparticle, sintering, densification, grain growth, two-sphere model 


\section{Introduction}

One of the manufacture means for nanocrystalline metals is to consolidate nanopowders by sintering or cold compression. For example, Nieman et al. [1] consolidated $\mathrm{Cu}$ and $\mathrm{Pd}$ nanopowders into nanocrystalline structures at room temperature using a pressure of $1.4 \mathrm{GPa}$, and Sanders et al. [2] warm compacted $\mathrm{Cu}$ and $\mathrm{Pd}$ nanopowder. Ragulya and Skorokhod [3] compared different sintering methods for Ni nanoparticles. Nanocrystalline metals are not the only outcome for nanoparticle sintering: by purposeful partial sintering of nanoparticles, porous structures can be obtained for various applications. Bai et al. [4] sintered Ag nanoparticles at a temperature as low as $280{ }^{\circ} \mathrm{C}$ and produced a microstructure containing micrometer-sized porosity. Nicula et al. [5] reported results concerning the synthesis of mesoporous titanium alloy bodies by spark plasma sintering. To assist and guide the above manufacturing techniques, an understanding on the atomic mechanism of nanoparticle sintering is crucial.

For micron-sized particles, a quite comprehensive body of theories for solid-state sintering has been developed. [6]The sintering process is divided into several stages. The first stage occurs when particles come into contact, since there is a weak cohesive bond at the contacts. The initial stage is accompanied by heating and is characterized by rapid neck growth between the adjoining particles. The intermediate and the final stages are marked by interconnected cylindrical pores and separate but nearly spherical pores, respectively. It is believed that shrinkage is low (less than 3\%) and grain growth is absent in the initial stage, and these mainly happen during the later stages. Six different mechanisms are believed to contribute toward the sintering of larger particles, namely,(1) surface diffusion,(2) lattice diffusion from surface,(3) vapour transport,(4) grain boundary diffusion,(5) viscous flow, and (6) plastic flow via dislocation movement. [6]

However, it has long been recognized that it is difficult to extrapolate conventional sintering theories developed for large particles to nanoparticles by using scaling laws[7]. For smaller particles, the effects of atomic forces are more significant, which may change the governing mechanisms for sintering altogether. Without using artificially assumed mechanisms, molecular dynamics is a useful tool for studying the sintering behaviour of nanoparticles, especially during the initial stage of contact adhesion during short time scales. To date, most MD studies on this topic were done to probe the sintering mechanism for two (or three) spheres 
during the initial stage. [8-23] Zhu and Averback [8] found the rotation of particles, together with the distortion and recrystallization of the neck region during sintering. They concluded that nanoparticle sintering involves a dislocation mechanism as the high shear stresses in the neck region exceed the theoretical strength of the material by applying Hertzian contact mechanics. The approach using two spheres is quite suitable for loosely packed nanoparticles sintered during gas phase production or on substrates. However, when nanoparticles are packed firmly, e.g. being consolidated into a nanocrystalline solid, the two-sphere model has the following shortcomings: (i) the important parameters for the sintered product, such as relative density and porosity, grain and pore sizes, etc., cannot be directly derived; (ii) the influence from pressure is not considered; (iii) there is no guarantee that the sintering mechanisms for two particles are the same for many particles. For instance, agglomeration, which is neglected in the two-sphere model, contributes significantly in the sintering rate. Very few reports were devoted to the MD simulations of the sintering of many nanoparticles. Kadau et al. [24] sintered firmly packed nanoparticles under a pressure of 1-2 GPa at a temperature of $600 \mathrm{~K}$ followed by relaxation in order to obtain nanocrystalline structures. Hawa and Zachariah [25] studied the sintering time for many nanoparticles arranged into a line shape and other shapes. In addition, Zhu and Averback [26] performed consolidation simulations on 2-D (hence infinitely long cylindrical) nanograins at temperatures ranging from $300 \mathrm{~K}$ to $1100 \mathrm{~K}$ and external pressures from 0 to $4 \mathrm{GPa}$, and found that a high initial packing density and external pressure enhance densification during sintering.

In order to perform a more comprehensive study on many-particle sintering, we simulated the sintering of copper nanoparticles under periodic boundary conditions at different temperatures and pressures. We also studied the influence of the initial particle size and spacing. We aim at comparing the MD results to the conventional solid-state sintering theories as well as the results from the two-sphere model.

\section{Simulation method}

We used periodic boundary conditions for the simulation box. Initially, randomly oriented nanoparticles of the same size were centred either on the corner or the face-centres of 
the cubic simulation box, to achieve a face-centred-cubic close-packing state of the particles. The size of the simulation box was set so that there was a small gap between each pair of adjacent particles. Constant external pressure was realized by controlling the dimensions of the cell using the Parrinello-Rahman method which is a widely used barostat strategy for MD simulations. [27] In this method, the coordinates of each atom $\left(\boldsymbol{r}_{i}\right)$ are represented as the sum of scaled coordinates $\left(\boldsymbol{s}_{\bar{i}}\right)$ and a matrix $\overline{\boldsymbol{h}}$ for the dimensions of the simulation box. In the present simulations, we restricted the shape of the box to be a cuboid with orthodox edges so that the simulation box matrix is given as

$$
\overline{\boldsymbol{h}}=\left|\begin{array}{ccc}
h_{x} & 0 & 0 \\
0 & h_{y} & 0 \\
0 & 0 & h_{z}
\end{array}\right| .
$$

The following equations then apply:

$$
\begin{aligned}
& \boldsymbol{r}_{i}=\overline{\boldsymbol{h}} \times \boldsymbol{s}_{i} \\
& \frac{d^{2} s_{i}}{d t^{2}}=-\overline{\boldsymbol{h}}^{-1} \frac{1}{m} \frac{\partial V}{\partial r_{i}}-2 \overline{\boldsymbol{h}}^{-1} \frac{d \bar{h}}{d t} \frac{d s_{i}}{d t} \\
& W \frac{d^{2} \bar{h}}{d t^{2}}=(-\boldsymbol{\sigma}-p \boldsymbol{I}) \overline{\boldsymbol{h}}^{-1} \operatorname{det}(\overline{\boldsymbol{h}})
\end{aligned}
$$

where $m$ and $V$ are the atomic mass and potential energy of the system respectively, $W$ is an empirical mass constant which determines how fast the size of the box can change, $p$ is the external hydrostatic pressure one wishes to maintain during the simulation, and $\boldsymbol{\sigma}$ is the virial stress given by

$$
\operatorname{det}(\overline{\boldsymbol{h}}) \boldsymbol{\sigma}=\Sigma_{i}-m v_{i} \otimes v_{i}+\Sigma_{j>i} \frac{\partial V}{\partial r_{i j}} \otimes r_{i j}
$$

where $v_{i}$ is the velocity of atom $i$.

However, oscillations in the box size are usually observed during equilibration of the system using the above scheme. A viscous damping term is usually added to the box's degrees of freedom to accelerate the speed of equilibration. Here, we employ a new algorithm to represent the damping term. This method has the following merits: (i) quick response of the system to 
maintain the desired pressure; (ii) the pressure of the system is always equal to or lower than the desired value to avoid overloading the structure; (iii) the evolution of the system is insensitive to the selection of $W$. All the other algorithms being the same as the above, and the following loop was added:

$$
\begin{aligned}
& \frac{d \bar{h}}{d t}=\int_{0}^{t} \frac{d^{2} h}{d t^{2}} d t, \text { if } \frac{d^{2} h}{d t^{2}} \cdot \frac{d \bar{h}}{d t}>0 \\
& \frac{d \overline{\boldsymbol{h}}}{d t}=0, \quad t=0 \text {, otherwise }
\end{aligned}
$$

The MD program employed made use of the leap-frog formulation with a time step of 2.5 fs. Atoms interacted through an EAM potential for $\mathrm{Cu}$ developed by Sheng et al. [28]. During the sintering simulations, the nanoparticles with no initial velocity were allowed to relax for 5ps (2,000 time steps) before the dimensions of the simulation box were allowed to change. The total simulation lasted for $0.25 \mathrm{~ns}$ (100,000 time steps). The velocity of each atom is scaled at each time step by the Woodcock thermostat control.

To facilitate the subsequent examination, after the sintering simulations, the resultants and mid-products were quenched to $0 \mathrm{~K}$ by using the conjugate gradient minimization technique. Unless specified otherwise, the resultants and mid-products shown below are quenched. To identify the existence of grain boundaries as well as other defects, the coordination number $(\mathrm{CN})$, i.e. the number of nearest neighbours of each atom was checked, and if an atom has $\mathrm{CN}$ exactly equal to 12 within a cut-off distance of $1.05 \times \mathrm{a}_{0} / \sqrt{ } 2$, we label it as an atom within a single crystal.

\section{Results}

\subsection{The sintering process of many nanoparticles}

Comparing with sintering between two spheres, a system with many nanoparticles has many more degrees of freedom. How nanoparticles are packed initially generally affects the densification behaviour during sintering. Particle packing coordination is used to describe the number of neighbours one particle has. Since each contact contributes concurrently to the loss of surface area, a high packing coordination number gives higher sintering rate. [6] It is a 
formidable task to test all the possible packing arrangements, and therefore we used an fcc closepacked arrangement for the initial nanoparticles in the simulations. Each particle in the system has 12 neighbours so that the green body density is at maximum value. This selection has its merits as well as drawbacks: under the assumption that nanoparticles are firmly and evenly packed, the densification rate derived in the simulations is actually the theoretical maximum value for real sintering. As for particle size, we used nanoparticles with number of atoms ranging from 531 to 75397 (diameter $=2.3-12.0 \mathrm{~nm})$. Four temperatures $(300 \mathrm{~K}, 500 \mathrm{~K}, 700 \mathrm{~K}, 900 \mathrm{~K})$ and three pressures $(0,0.25 \mathrm{GPa}$ and $2 \mathrm{GPa})$ were employed in the different simulation cases. Meanwhile, we also investigated the influence of the interparticle gap. Although the packing arrangement was always set to be fcc-close-packing, the gap between the particles, i.e. the closest distance between the surfaces of two nearest-neighbour particles, can be altered. We set the interparticle gap to be 4,5 or $6 \AA$ Á.

Before the sintering, we examined whether the nanoparticles will go through phase or structural transition at the given temperature. For instance, at temperature $=900 \mathrm{~K}$, for particles with size equal to or under $3.4 \mathrm{~nm}$ bulk or surface melting occur when equilibrated individually. The ones that are not stable were ruled out for the subsequent simulations. For sintering, we placed 4 randomly oriented nanoparticles of the same size in the simulation box with periodic boundary conditions as shown in Fig. 1(a). After relaxation, rapid densification occurred. The size of the simulation box was allowed to vary in response to the system pressure. The density of the system served as an indicator for densification when compared with the full density of the fcc crystalline state of copper at the same temperature and pressure. It is found that fast densification generally occurs during the first 100ps of simulations, and the shrinkage of simulation box is negligible after 100ps. At the end of the simulation, three possible outcomes appear:

1) All pores disappear and full densification is reached at the end of the simulation (Fig. 1(b)). Note that the sintered product is seldom perfect fcc crystal but generally carries defects such as vacancies, twinning, stacking faults and dislocation loops. In Fig. 2(a) a dislocation loop is plotted, and twinning is shown in Fig. 2(b). Vacancies can be identified by the $\mathrm{CN}$ checking method specified in the previous section.

2) The pores between particles become disconnected and quasi-spherical. The pore shrinkage is significant, but full densification is not reached (Fig. 1(c)). 
3) For very small nanoparticles (diameter $<3 \mathrm{~nm}$ ), sometimes large pores with diameters $>0.65 \mathrm{~nm}$ remain in the sintered product. In some cases, two crystalline parts are completely detached from each other (Fig. 1(d)). The reason is that the gap becomes wider than the cutoff distance of $0.65 \mathrm{~nm}$ in the EAM potential, once such a wide gap is formed the separated parts will no longer interact. The porosity at the end of the simulations is usually larger than 0.2. This phenomenon corresponds to the agglomeration of particles in real sintering.

Showing the evolution history may shed light on the sintering mechanism. For illustration, we use snapshots taken from one case, where the number of atoms in each nanoparticle is 531 , temperature is $300 \mathrm{~K}$ and no external pressure is exerted, and initial interparticle gap is set to be $4 \AA$ (Fig. 3). Although initially 4 particles in the simulation box are dispersed evenly, each pair of particles are not approaching each other at the same rate after the relaxation due to the atomic degrees of freedom. As a result, some gaps are eliminated more quickly than others. When two nanoparticles bond with each other, their crystal orientations undergo rotations. A large proportion of the crystal, especially the region near the neck, appears amorphous. Rapid recrystallization takes place shortly afterwards, as revealed in both the crystal structure plots and the simulated diffraction patterns at the lower panel of Fig. 3. For this case, the sintered product is an fcc crystal with a few defects. For other cases, nanocrystalline structures remain at the end. Details on grain growth will be discussed in Section 3.3.

To quantify the mobility of atoms during the sintering of many nanoparticles, we calculated the mean squared displacement of the atoms during sintering. The mean square displacement (MSD) is a measure of the average distance that a given particle in a system travels:

$$
\operatorname{MSD}=\left\langle r^{2}(t)\right\rangle=\left\langle\frac{1}{N} \sum_{i=1}^{N}\left(r_{i}(t)-r_{i}(0)\right)^{2}\right\rangle
$$

Here $N$ is the number of atoms in the system, $r_{i}(t)$ and $r_{i}(0)$ are the coordinates of atom $i$ at time $t$ and time 0. Fig. 4 presents the MSD evolution for $3.4 \mathrm{~nm}$ nanoparticles sintered under different temperatures and zero pressure. The slope of the curves can serve as a measurement of an averaged migration speed of the equilibrium positions of atoms. 
For micron sized particles, diffusion is generally regarded as the key process during sintering. Among all the diffusion forms, surface diffusion is the fastest kind. A typical surface diffusion event is depicted in the following: first of all an atom breaks away from the bonding site, then moves with a random motion across the surface, and finally it reattaches to another site [6]. Apparently the mobility of surface atoms must be upper-bounded by the rate of the second step, which is stochastic surface diffusion. We calculated the surface diffusivity of copper adatoms on (100) plane according to

$$
D_{T h}=\frac{1}{2 m} a^{2} v e^{\frac{-\unlhd E}{k_{B} T}}
$$

where $m$ is the dimensionality of the system which, for (100) plane surface diffusion is $2, v$ is the characteristic thermal vibration frequency of the material, and for metals, this is around $10^{12} \sim 10^{13} \mathrm{~Hz}, a$ is the length of each diffusion jump, and is $a_{0} / \sqrt{2}$ in this case, and $\Delta E$ is the activation energy (i.e. energy difference of the transition and equilibrium states). Here, the entropy contribution to the diffusivity in eqn. (7) is ignored because the atomic configurations of the transient and equilibrium states are fixed so that the configurational entropy for both states is zero although the vibrational entropy is not, and moreover, the entropy term is usually ignored in DFT calculations [ref.]. Using the present EAM potential, $\Delta E$ is calculated to be $0.493 \mathrm{eV}$. Based on these values, the surface diffusivities of copper atoms on (100) plane at different temperatures are listed in Table 1. As adatoms undergo 2-D random walk, the theoretical mean square displacement of all atoms is bounded by the surface diffusivity in the following way:

$$
\left\langle r_{t h}^{2}\right\rangle<4 t D_{t h}
$$

If diffusion is the dominating mechanism of the system's densification, then we would expect the above mentioned slope of MSD curves to be less than $4 D_{\text {th }}$ at the system temperature and it should be very sensitive to temperature. But from Fig. 4, the slopes of the curves at the beginning of the simulations, roughly during the first $50 \mathrm{ps}$, amount to more than $5 \times 10^{-4} \mathrm{~cm}^{2} / \mathrm{s}$, which is several orders larger than $4 D_{t h}$ at the system temperature. Besides, the negative slopes in some region do not accord with the characteristics of random walks. Furthermore, the slopes are not sensitive to the temperature. To sum up, the densification of particles observed from the simulations happens at much faster speeds than what surface 
diffusion can allow, i.e. diffusion cannot be the dominating mechanism for the densification process in our MD simulations. Some other mechanisms must govern this densification process. At high temperatures $\left(T \sim T_{m}\right)$, diffusion is certainly not negligible, but as our simulations are performed at lower temperatures $(\mathrm{T} \leq 900 \mathrm{~K})$, the role of diffusion is not significant.

On the other hand, vapour transport and viscous flow mechanisms suggested by the conventional sintering theory are not activated either. Although the melting temperature for nanoparticles is significantly lower than the bulk one, at the temperature below $900 \mathrm{~K}$, the particles used for the simulations did not undergo bulk or surface melting when equilibrated separately. Plastic flow mechanism was proposed for materials with dislocations before sintering, and therefore this is not applicable to nanoparticles that are initially defect-free. Having ruled out these conventional mechanisms, we suspect that densification is due to the high stress in the neck region causing the material to deform plastically by either dislocation or twinning mechanisms, or even by viscous flow following amorphization. Such plasticity mechanisms can explain the large MSD and the amorphous region at the neck at the early stage of sintering.

\subsection{Influence from various factors on densification process}

First of all, we present the influence from particle size and temperature on sintering. We performed four sets of simulations at zero external pressure and the temperature of $300 \mathrm{~K}, 500 \mathrm{~K}$, $700 \mathrm{~K}$ or $900 \mathrm{~K}$. For each set of simulations, nanoparticles with size ranging from $2.3 \mathrm{~nm}-12 \mathrm{~nm}$

were used. The initial interparticle gap was $4 \AA$. The relative density of the sintered product is defined as the ratio of real density divided by the theoretical density of perfect copper fcc crystal at the same conditions. Porosity is unity minus relative density. The porosity of different systems with different initial particle size after being sintered for $250 \mathrm{ps}$ is shown in Fig. 5. The trend clearly suggests that smaller initial particle size and higher temperature promote densification as the residual porosity at $250 \mathrm{ps}$ remains low. It should be noted that if the porosity is less than 0.015, there is no noticeable pore in the system, i.e. the system is effectively fully densified. Notice that the porosity for a fully densified system is not exactly zero because of the existence of defects (e.g. grain boundaries, dislocations, vacancies, etc.). For larger particle sizes and at 
lower temperatures, the residual porosity can remain to be a few $\%$ or higher, and in these cases the system is not fully densified. For all the simulation cases except two, agglomeration did not occur. The illustration of agglomerated products is in Fig. 1(d).

From experimental results, it is known that hydrostatic pressure facilitates densification. To analyze this effect, we performed two sets of simulations under the external pressure of $0.25 \mathrm{GPa}$ or $2 \mathrm{GPa}$. The temperature was $300 \mathrm{~K}$ and the interparticle gap was set to be $4 \AA$. The results are shown in Fig. 6, together with reproduced results from zero pressure sintering for the sake of comparison. It can be seen that a high pressure (2GPa) facilitates densification while the effect from low pressure $(0.25 \mathrm{GPa})$ is far less pronounced. However, when we applied the low pressure $(0.25 \mathrm{Gpa})$ on some simulation cases where agglomeration will occur under zero pressure, the phenomenon of agglomeration was absent.

The influence of interparticle gap was also investigated. Three sets of simulations were performed on nanoparticles of various sizes under different particle spacing at $300 \mathrm{~K}$ and no external pressure. The interparticle gap for each set of simulations was about 4,5 or $6 \AA \AA$, and the results are shown in Fig.7. In four cases agglomeration occurred, all for very small nanoparticles. For other cases, the three curves almost overlap, indicating that interparticle gap has little effect on the densification process, as long as agglomeration does not occur. Larger gap seems to promote agglomeration only when the particle size is sufficiently small.

\subsection{Grain growth}

Grain growth in nanoparticle sintering cannot be ignored because this can cause the product to lose nanocrystalline characteristics. In Fig. 3 we have shown how four nanoparticles with different orientations may evolve into a single fcc crystal. For the other simulation cases in the present work, grain growth also extensively exists. In the present work, since the system only contains four grains to start with, the grain size can only take a set of limited and discrete values. Hence, the grain sizes at the end of the sintering simulations are not regarded as an accurate indicator for the grain growth rate. Instead, we analyzed our results qualitatively based on the number of grains in each simulation box at the end of sintering as well as the movement of grain boundaries. We classified the outcomes into three categories: (i) There is only one grain left in 
the simulation box, i.e. the sintered product is a single crystal (Fig. 8(a)). From the simulations, the single crystal at the end is seldom defect-free, as most of them carry one or more dislocation loops, and twinning and stacking faults are often observed. The annihilation of grain boundaries usually happens for very small nanoparticles (diameter $<4 \mathrm{~nm}$ ) and is possible for nanoparticles with intermediate sizes $(4 \mathrm{~nm}<$ diameter $<6 \mathrm{~nm})$. (ii) There are still 4 grains in the simulation box (Fig. 8(b)), and the locations of the grain boundaries remain still during sintering. This usually happens for larger nanoparticles (diameter $>6 \mathrm{~nm}$ ). (iii) There are more than one grain in the box (Fig. 8(c)), and the shape of the grains is highly distorted and far from spherical. The locations of the grain boundaries also vary significantly from their initial locations. This often occurs for nanoparticles with intermediate sizes $(4 \mathrm{~nm}<$ diameter $<6 \mathrm{~nm})$. This outcome can be regarded as the mid-way between the previous two situations. We found that pressure and interparticle gap have little effect on grain growth. Higher temperature facilities grain growth, but its influence is far less pronounced than that from particle size.

We are interested in how the grain boundaries are moved or annihilated during the sintering process. Fig. 9 shows snapshots of grain boundaries during the sintering of nanoparticles each of 1253 atoms at $300 \mathrm{~K}$, with the sintered product illustrated in Fig. 8(a). The snapshots show that the grain boundaries move radically and chaotically instead of gradually in a coordinated manner. Grain growth activity is concentrated at the first 100 ps of the sintering, simultaneously with the densification. The number of amorphous atoms (i.e. atoms with less than $12 \mathrm{CN}$ ) first increases then drops. This suggests that the grain growth during the sintering simulations is not through the conventional grain boundary migration mechanism. In Fig. 3, the rotation and sliding of grains are revealed, which may be responsible for the grain growth.

\subsection{Comparison with two-sphere model}

Most past works on the sintering of nanoparticles have focussed on the behaviour of two spherical particles. There is little justification of whether the result from such a two-sphere model can be extended to the case of many nanoparticles sintering. To fill this gap, we examined whether the results from the two-sphere sintering model and those from many nanoparticles sintering are consistent. Although two-sphere sintering has been simulated in a number of 
previous reports, no comprehensive data exists for different sized copper nanoparticles sintered at different temperatures. Therefore, we performed sintering simulations between two spherical copper nanoparticles of the same size. The initial gap between two particles was always set to be $4 \AA$ Á. After relaxation, the two particles move closer as indicated by shrinkage. In some studies, shrinkage was defined as $\frac{\Delta L}{L_{0}}$, where $L_{0}$ is the distance between the centres of mass of the initial nanoparticles and $\Delta L$ is the change in this distance. Here, we used a slightly different definition:

$$
\text { shinkage }=\left(d-d_{\text {CoM }}\right) / d
$$

where $d$ is the diameter of the initial nanoparticles which is really $L_{0}$ minus the gap size, while $d_{\mathrm{COM}}$ is the distance between their real-time centres of mass.

We are primarily interested in the sintering behaviour at the low temperature regime. We carried out the sintering simulations for particles with various sizes and crystal orientations at $300 \mathrm{~K}$ and recorded the shrinkage at 500ps. This time interval is selected because shrinkage is always stabilized within 100ps. Note that the shrinkages for particles with different orientations are generally different. For this reason, we did 4 simulation cases for each group of nanoparticles with the same size.

From Fig. 10, it can be seen that shrinkage is declining approximately inverse proportionally with respect the particle size at $300 \mathrm{~K}$. We are aiming at comparing the densification in many nanoparticle sintering with shrinkage value obtained in two-sphere sintering simulations. To do that, we assume that each dimension of the simulation box shrinks isotropically, so that the densification can be expressed as:

$$
\text { densification }=0.74 \times(1-\text { shrinkage })^{-3}
$$

Here, 0.74 is the relative density of closely packed spheres comparing with bulk density. Since it is impossible for the densification to exceed unity, the value from the above expression is capped to 1 , viz,

$$
\text { porosity }=\max \left(1-0.74 \times(1-\text { shrinkage })^{-3}, 0\right)
$$


In Fig. 11 we plot the theoretical porosity calculated from the shrinkage of the two sphere sintering simulations and the porosity derived from many-particle sintering simulations. The data from the two situations agree satisfactorily.

From the many-particle sintering simulations, we learned that higher temperature and pressure eliminate the porosity of the sintered product. It is difficult to incorporate pressure in the two particle sintering case, but the effect from temperature can be studied. In Fig. 12, shrinkages are presented for two nanoparticles with same orientation at different temperatures. The sintering behaviour is drastically different for a low temperature regime $(T \leq 900 \mathrm{~K})$ and a high temperature regime ( $T>900 \mathrm{~K})$. For the low temperature regime, the shrinkage, which is much smaller than that for the high temperature regime, is stabilized after the first $50 \mathrm{ps}$. The shrinkage drops as temperature rises, however the dependence is weak. For the high temperature regime ( $T$ $>900 \mathrm{~K}$ ), the shrinkage drops drastically during the first 100ps and continues to decrease, albeit at a lower rate, throughout the whole time span of the simulations. Besides plastic deformation, we think additional mechanisms may contribute to the high temperature sintering behaviour such as diffusion and viscous flow. As listed in Table 1, surface diffusion rate at high temperature is considerable. Bulk and surface melting of the particles suggests the presence of viscous flow as a mass transport mechanism.

In two-sphere sintering simulations, it is rather difficult to obtain conclusions on grain growth. The only indicator is whether two particles in the end of the simulation are aligned with no grain boundary in between. It is found that for nanoparticles with diameter of less than $3.5 \mathrm{~nm}$, the annihilation of grain boundary is very common during sintering simulations at $300 \mathrm{~K}$ in the present work. Small misorientation of two particles also contributes to this phenomenon.

Overall, the data from many-particle and two-sphere sintering agree well, indicating that the overall densification of many particles is really governed by the sintering of each pairs of neighbouring particles, provided that agglomeration is absent. It is reasonable to conclude that the main mechanisms for sintering are the same in both cases.

For two-sphere sintering, it is possible to calculate the stress at the neck. From Fig. 13, the normal stress at the neck average from time $=5-10 \mathrm{ps}$ peaks at around $10 \mathrm{GPa}$ for two nanoparticles sintered at $300 \mathrm{~K}$, which is comparable with the theoretical strength of $\sim E / 10$ where 
$E$ is Young's modulus. Provided that the diffusion and viscous flow mechanisms are not activated at such a low temperature, the high stress in the neck region is the governing factor for the rapid shrinkage.

The high stresses at the neck make the particles deform plastically by generating defects such as dislocations and deformation twinning during two-sphere sintering. To reveal dislocations and twins, we make use of relative displacement plots to show how atoms in the initial particles are displaced relative to their nearest-neighbours at the end of the simulation. In Fig. 14, we plot the atoms in one layer of [100] plane crossing the centre of one of the initial particles before sintering in blue colour. The initial particle under analysis is the left one of the sintering pair. For each pair $(i, j)$ of nearest-neighbour atoms, their relative displacement $r_{i j}$ is the displacement of atom $i$ relative to that of atom $j$. To distinguish the direction of $r_{i j}$, we define atom $i$ to be always on the right hand side of atom $j$, or when they have the same horizontal coordinate, atom $i$ to be above atom $j$. In Fig. 14, the in-plane component of $r_{i j}$ is represented in both magnitude and direction by a black arrow drawn between each atomic pair, and the out-ofplane component of $r_{i j}$ is represented by a vertical red arrow that points upward if the out-ofplane component of $r_{i j}$ is out of the paper and downward if into the paper. Note that the rotations of the particles are subtracted for easier interpretation. In Fig. 14(a), with the trace produced by the cross-slip of a dislocation is revealed on the right side of the particle at the contact region. Dislocation slip is extensively observed for nanoparticles with larger sizes (diameter $>4 \mathrm{~nm}$ ) during sintering simulations. Fig. 14(b) illustrates a twin in one sintered product. Each successive $\{111\}$ plane in the twinned region is displaced by a shear vector approximately the same as the Shockley partial (i.e. the difference is due to the strain in the structure). Twinning usually happens for very small particles (diameter $<4 \mathrm{~nm}$ ). Notice that a few vectors at the right hand side of the particle, i.e. at the contact neck, appear irregular. A possible explanation is that small scale amorphization occurred at the contact neck during sintering.

\section{Discussion}

In a number of previous studies on MD simulations, it has been proposed that the

mechanism for the sintering of nanoparticles is different from that for larger particles. Zhu and Averback [8] concluded that a dislocation mechanism is mainly responsible for the sintering of 
copper nanoparticles at $700 \mathrm{~K}$ as the stress in the neck region exceeds the theoretical strength of the material. Liu et al. [29] also suggested that at low temperatures, coalescence of nanoparticles is due to the high shear stresses developed at the contact regions between the particles. Song and Wen [21] concluded that surface diffusion and grain boundary diffusion, the contribution of viscous flow, plastic deformation, and surface tension gradient are important in the sintering process. They also found a higher mobility for surface atoms than bulk atoms during sintering. Our conclusions generally agree with some of the above mentioned findings; in particular, high stresses similar to the theoretical strength, and dislocation glide and twinning for the two-particle case, were observed at the neck region. However, by comparing the apparent diffusion coefficient and theoretical diffusivity of the copper atoms, we concluded that diffusion cannot be a significant contributing factor for the rapid shrinking at the early stage of sintering at low temperature regime $(\mathrm{T} \leq 900 \mathrm{~K})$. The governing mechanism that we have identified is also different from other conventional sintering mechanisms suggested [6], including vapour transport and plasticity due to pre-existing dislocations: no gas phase was observed in the present simulations, indicating that vapour transport is absent, and the initial particles in the present simulations do not containing pre-existing dislocations. Besides, the present simulated results for nanoparticles do not show division of the sintering process into distinctive "stages" as assumed in the conventional theory for larger particles. [6]

During many nanoparticle sintering simulations, we observed both dislocation and twinning plasticity mechanisms (Fig. 2), as well as amorphization (Fig. 3) near the contact neck regions of the nanoparticles. Such phenomena are unaccounted for by the conventional mechanisms. Although it is difficult to determine the stresses at the neck regions in the manyparticle simulations, we envisage that, as is similar to the two-particle situation, the stress levels there for the many-particle case also exceed the theoretical strength of the material and hence cause the various plasticity processes mentioned above. The fact that the shrinkage rate of the many-particle sintering can be well described by the two-particle model (Fig. 11) also supports that the critical mechanisms at the contact regions are the same in the two situations. In summary, therefore, the sintering of nanoparticles, whether in many-particle or two-sphere settings, is controlled by a variety of plasticity mechanisms at the contact neck regions between particles, including dislocation, twinning and amorphization, and these are due to the high stresses there. 


\section{Conclusions}

We investigated the sintering behaviour of closely-packed many nanoparticles at low temperature regime ( $\mathrm{T} \leq 900 \mathrm{~K})$. The governing sintering mechanism is found to be a variety of plasticity mechanisms, including dislocation, twinning and amorphization, due to the high stress levels near the neck regions. Without agglomeration, smaller particle sizes facilitate densification along with higher temperature and external pressure, while interparticle gap exerts only a mild influence. It is also found that drastic grain growth appears for nanoparticles of very small sizes (D $<6 \mathrm{~nm}$ ). A two-sphere sintering model also shows good agreement with the many-particle sintering simulations.

\section{References}

[1] G.W. Nieman, J.R. Weertman, R.W. Siegel, Journal of Materials Research, 6:5 (1991) 1012-1027.

[2] P.G. Sanders, A.B. Witney, J.R. Weertman, R.Z. Valiev, R.W. Siegel, Materials Science and Engineering: A, 204 (1995) 7-11.

[3] A.V. Ragulya, V.V. Skorokhod, Nanostructured Materials, 5 (1995) 835-843.

[4] J.G. Bai, Z. Zhiye Zach, J.N. Calata, L. Guo-Quan, Components and Packaging Technologies, IEEE Transactions on, 29 (2006) 589-593.

[5] R. Nicula, F. Lüthen, M. Stir, B. Nebe, E. Burkel, Biomolecular Engineering, 24 (2007) 564-567.

[6] R.M. German, Sintering Theory and Practice, John Wiley \& Sons, Inc., 1996.

[7] C. Herring, Journal of Applied Physics, 21 (1950) 301-303.

[8] H. Zhu, R.S. Averback, Philosophical Magazine Letters, 73 (1996) 27-33.

[9] L.J. Lewis, P. Jensen, J.-L. Barrat, Physical Review B, 56 (1997) 2248-2257.

[10] J.S. Raut, R.B. Bhagat, K.A. Fichthorn, Nanostructured Materials, 10 (1998) 837-851.

[11] P. Zeng, S. Zajac, P.C. Clapp, J.A. Rifkin, Materials Science and Engineering: A, 252 (1998) 301-306.

[12] K.E.J. Lehtinen, M.R. Zachariah, Physical Review B, 63 (2001) 205402.

[13] S. Hendy, S.A. Brown, M. Hyslop, Physical Review B, 68 (2003) 241403.

[14] F. Ding, A. Rosén, K. Bolton, Physical Review B, 70 (2004) 075416.

[15] T. Hawa, M.R. Zachariah, Journal of Aerosol Science, 37 (2006) 1-15.

[16] I. Karkin, L. Karkina, Y.N. Gornostyrev, Materials Science Forum, Volumes 584 - 586 (2008) 1033-

1038.

[17] H. Pan, S.H. Ko, C. P.Grigoropoulos, The Solid-State Neck Growth Mechanisms in Low Energy Laser Sintering of Gold Nanoparticles : A Molecular Dynamics Simulation Study, American Society of Mechanical Engineers, New York, NY, ETATS-UNIS, 2008.

[18] L. Ding, R.L. Davidchack, J. Pan, Computational Materials Science, 45 (2009) 247-256.

[19] H.H. Kart, G. Wang, I. Karaman, T. Cagin, International Journal of Modern Physics C, Vol. 20 (2009) 179-196.

[20] A. Moitra, S. Kim, S.-G. Kim, S.J. Park, R.M. German, M.F. Horstemeyer, Acta Materialia, 58 (2010) 3939-3951.

[21] P. Song, D. Wen, Journal of Nanoparticle Research, 12 (2010) 823-829. 
[22] J. Antúnez-García, S. Mejía-Rosales, E. Pérez-Tijerina, J. Martín, Montejano-Carrizales, M. JoséYacaman, Materials, (2011) 368-379.

[23] L. Yang, Y. Gan, Y. Zhang, J. Chen, Applied Physics A: Materials Science \& Processing, 106 (2012) 725-735.

[24] K. Kadau, P. Lomdahl, B. Holian, T. Germann, D. Kadau, P. Entel, D. Wolf, M. Kreth, F. Westerhoff, Metallurgical and Materials Transactions A, 35 (2004) 2719-2723.

[25] T. Hawa, M.R. Zachariah, Journal of Aerosol Science, 38 (2007) 793-806.

[26] H. Zhu, R.S. Averback, Materials Science and Engineering: A, 204 (1995) 96-100.

[27] M. Parrinello, A. Rahman, Journal of Applied Physics, 52 (1981) 7182-7190.

[28] H.W. Sheng, M.J. Kramer, A. Cadien, T. Fujita, M.W. Chen, Physical Review B, 83 (2011) 134118.

[29] H.B. Liu, M. José-Yacaman, R. Perez, J.A. Ascencio, Applied Physics A: Materials Science \&

Processing, 77 (2003) 63-67.

Table 1 The surface diffusivity of copper atoms on (100) plane at different temperatures

\begin{tabular}{|c|c|}
\hline Temperature (K) & Surface Diffusivity $\left(\mathrm{cm}^{2} / \mathrm{s},\right)$ \\
\hline 300 & $9 \times 10^{-12}$ \\
\hline 500 & $2 \times 10^{-8}$ \\
\hline 700 & $5 \times 10^{-7}$ \\
\hline 900 & $3 \times 10^{-6}$ \\
\hline 1100 & $9 \times 10^{-6}$ \\
\hline 1300 & $2 \times 10^{-5}$ \\
\hline
\end{tabular}




\section{Figure Captions}

Fig. 1 The sintered configuration of the system of different cases: (a) before sintering; (b) a sintered product at full densification; (c) a sintered product when partially densified; (d) a sintered product with large pores. Atoms from different initial particles are coloured differently.

Fig. 2 Crystal structures of sintered products: (a) a fully densified product with a dislocation loop; (b ) a fully densified product with twinning. Twin boundaries are marked by red lines.

Fig. 3 Snapshots of crystalline structures at different stages of sintering. Time and porosity are indicated below each panel. Simulated powder diffraction diagrams are provided at the lower panel.

Fig. 4 MSD for $3.4 \mathrm{~nm}$ nanoparticles sintered at different temperatures.

Fig. 5 Porosity of the sintered products with different particle size and temperature. The cases with agglomerations are circled out in the graph.

Fig. 6 Porosity of the sintered products under different pressures.

Fig. 7 Porosity of the sintered products with different initial interparticle gaps. The cases with agglomerations are circled out in the graph.

Fig. 8 Grain boundaries in the sintered products. Atoms on grain boundaries and other defects, i.e. those with less on $12 \mathrm{CN}$, are labelled red, and atoms on fcc lattice, i.e. those with $12 \mathrm{CN}$, are labelled blue.

Fig. 9 Snapshots of grain boundaries during sintering. Atoms on grain boundaries and other defects, i.e. those with less on $12 \mathrm{CN}$, are labelled red, and atoms on fcc lattice, i.e. those with 12 $\mathrm{CN}$, are labelled blue. Time is indicated below each snapshot.

Fig. 10 Shrinkage of two nanoparticles sintered at 300K. 
Fig. 11 Porosity derived from two-sphere model and many particle sintering simulations. Red dot: theoretical porosity calculated from two sphere shrinkage. Black line: porosity from many particle sintering simulations. The temperature of the system is $300 \mathrm{~K}$.

Fig. 12 Shrinkage with respect to time for two $6.0 \mathrm{~nm}$ nanoparticles with same orientation sintered at different temperatures.

Fig. 13 Average radial distribution of normal stresses at neck during sintering of two nanoparticles each of 10005 atoms at $300 \mathrm{~K}$.

Fig. 14 Relative displacement maps for typical sintered products at the end of the simulation.

Fig. 1 The sintered configuration of the system of different cases: (a) before sintering; (b) a sintered product at full densification; (c) a sintered product when partially densified; (d) a sintered product with large pores. Atoms from different initial particles are coloured differently.

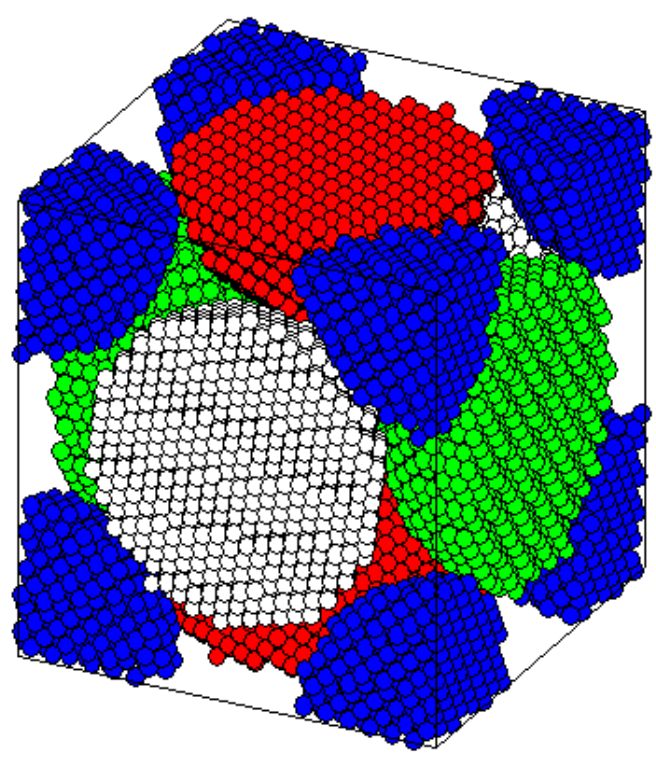

(a)

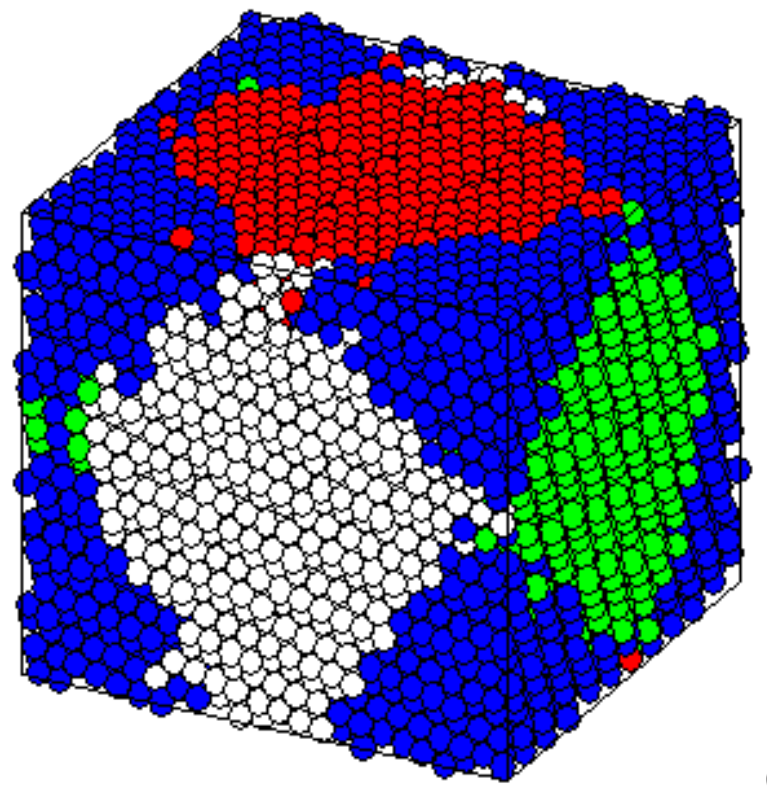

b) 


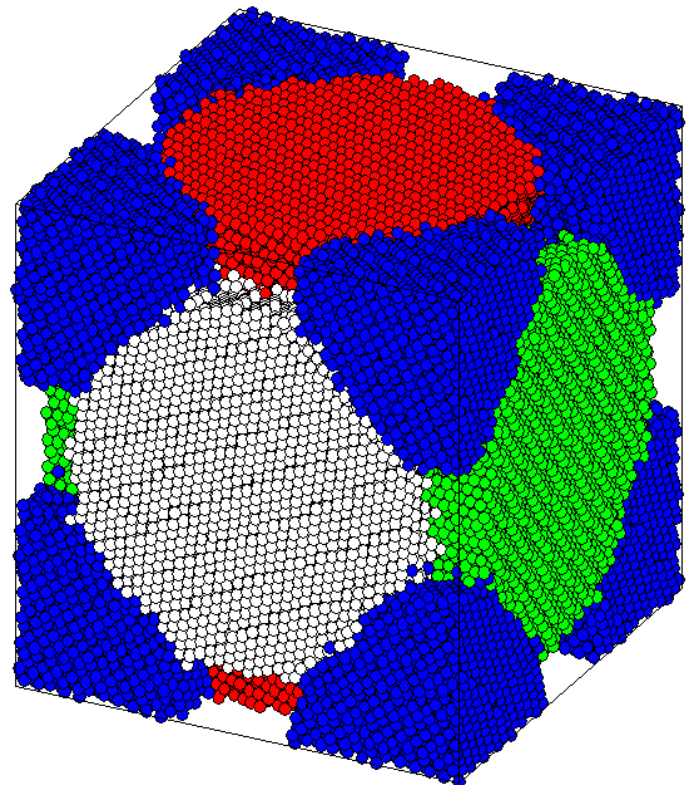

(c)

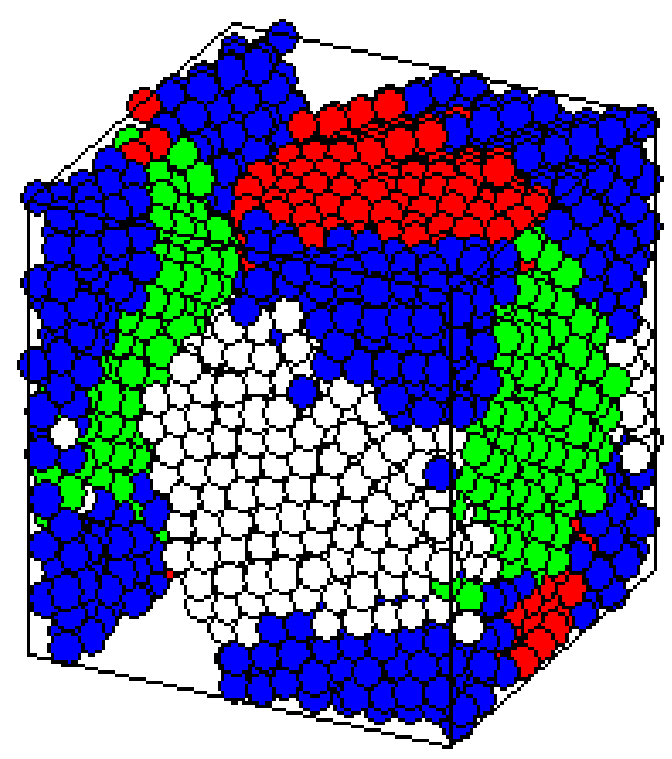

(d)

Fig. 2 Crystal structures of sintered products: (a) a fully densified product with a dislocation loop; (b ) a fully densified product with twinning. Twin boundaries are marked by red lines.

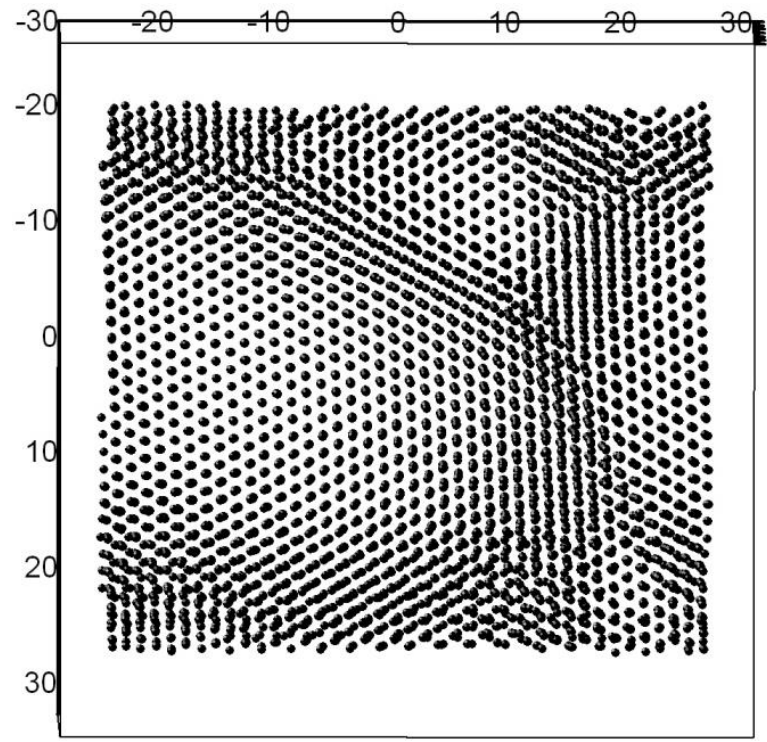

(a)

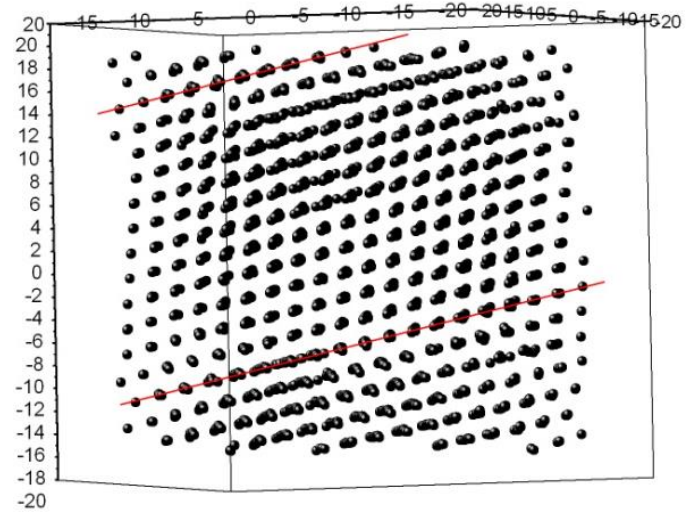

(b) 
Fig. 3 Snapshots of crystalline structures at different stages of sintering. Time and porosity are indicated below each panel. Simulated powder diffraction diagrams are provided at the lower panel.
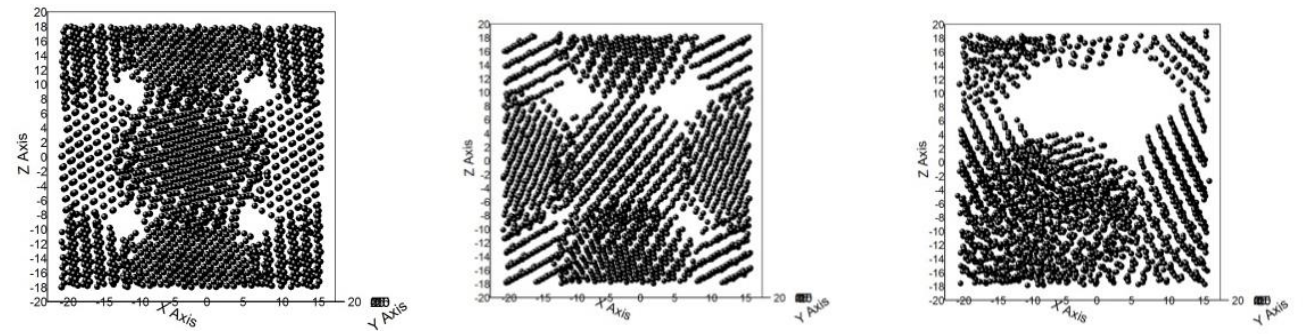

$t=0 \mathrm{ps}$ porosity: 0.46

$$
t=5 \text { ps porosity: } 0.46
$$

$t=10$ ps porosity:0.45
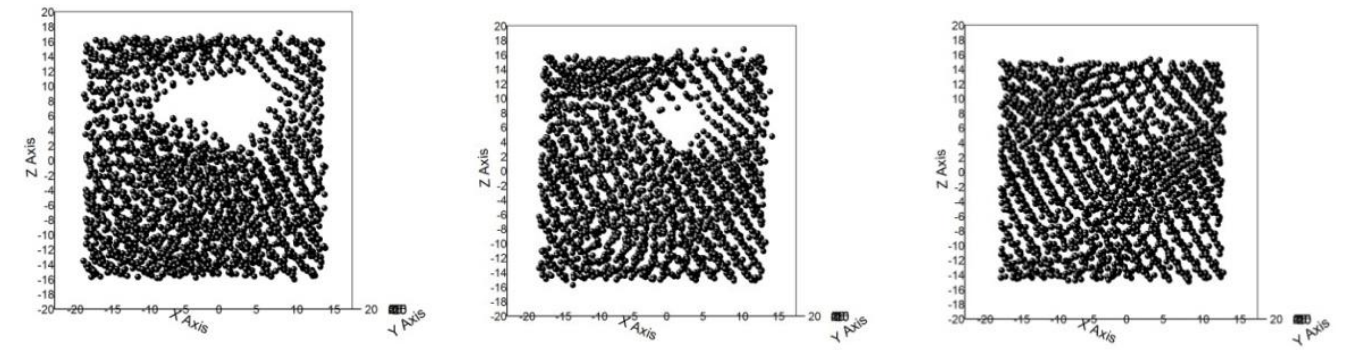

$t=25$ ps porosity:0.22

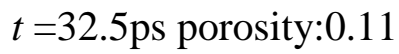

$t=37.5$ ps porosity $=0.05$ 

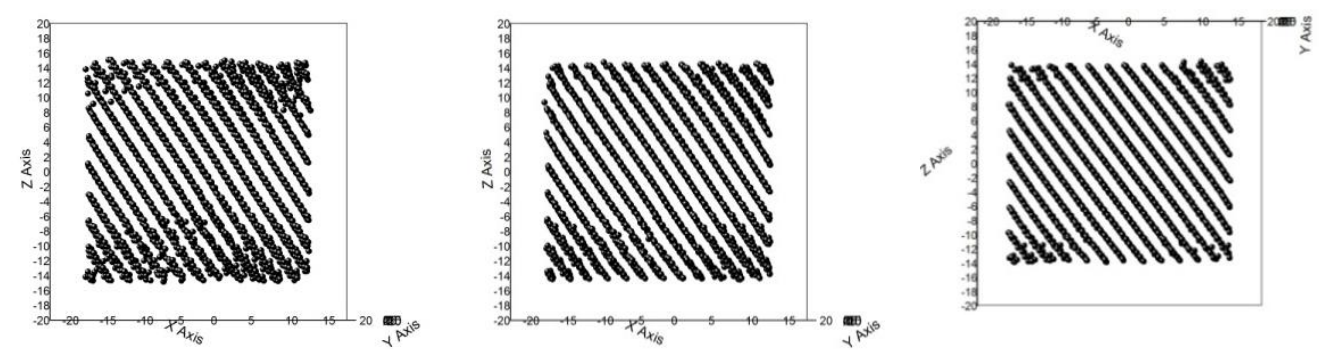

$t=42.5$ ps porosity: 0.01

$t=50$ ps porosity: 0.01

$t=250$ ps porosity: 0.01

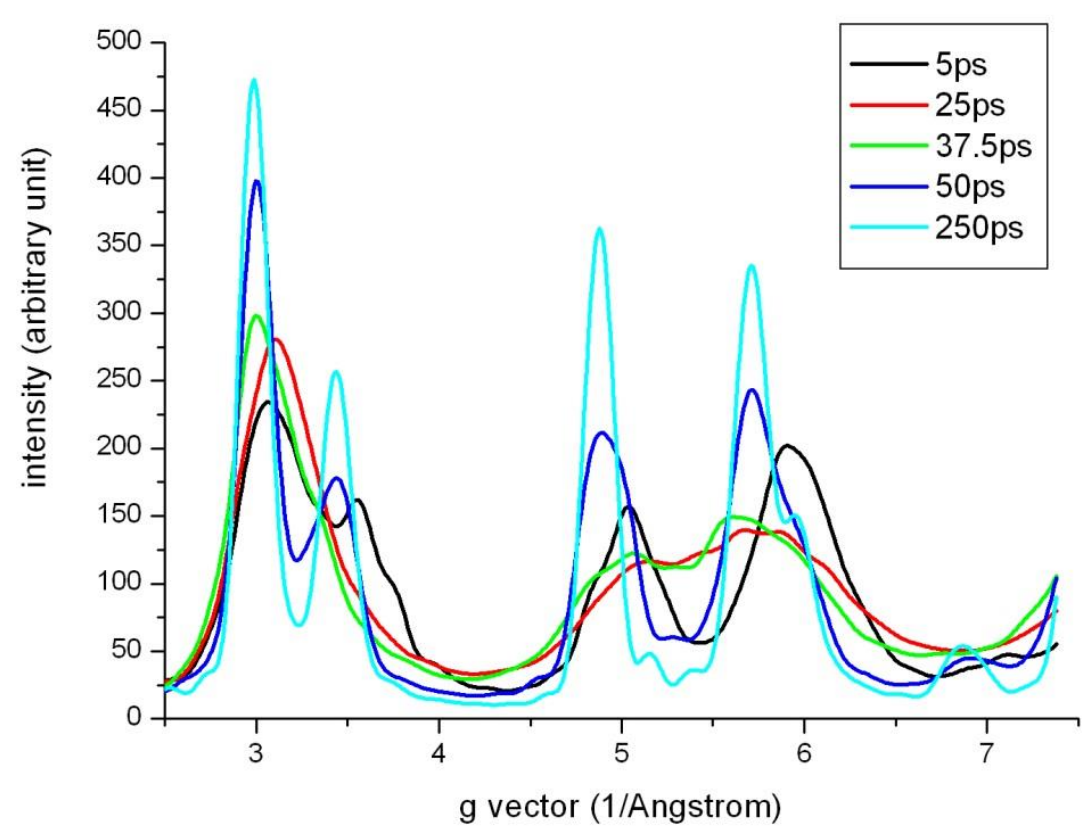


Fig. 4 MSD for $3.4 \mathrm{~nm}$ nanoparticles sintered at different temperatures.

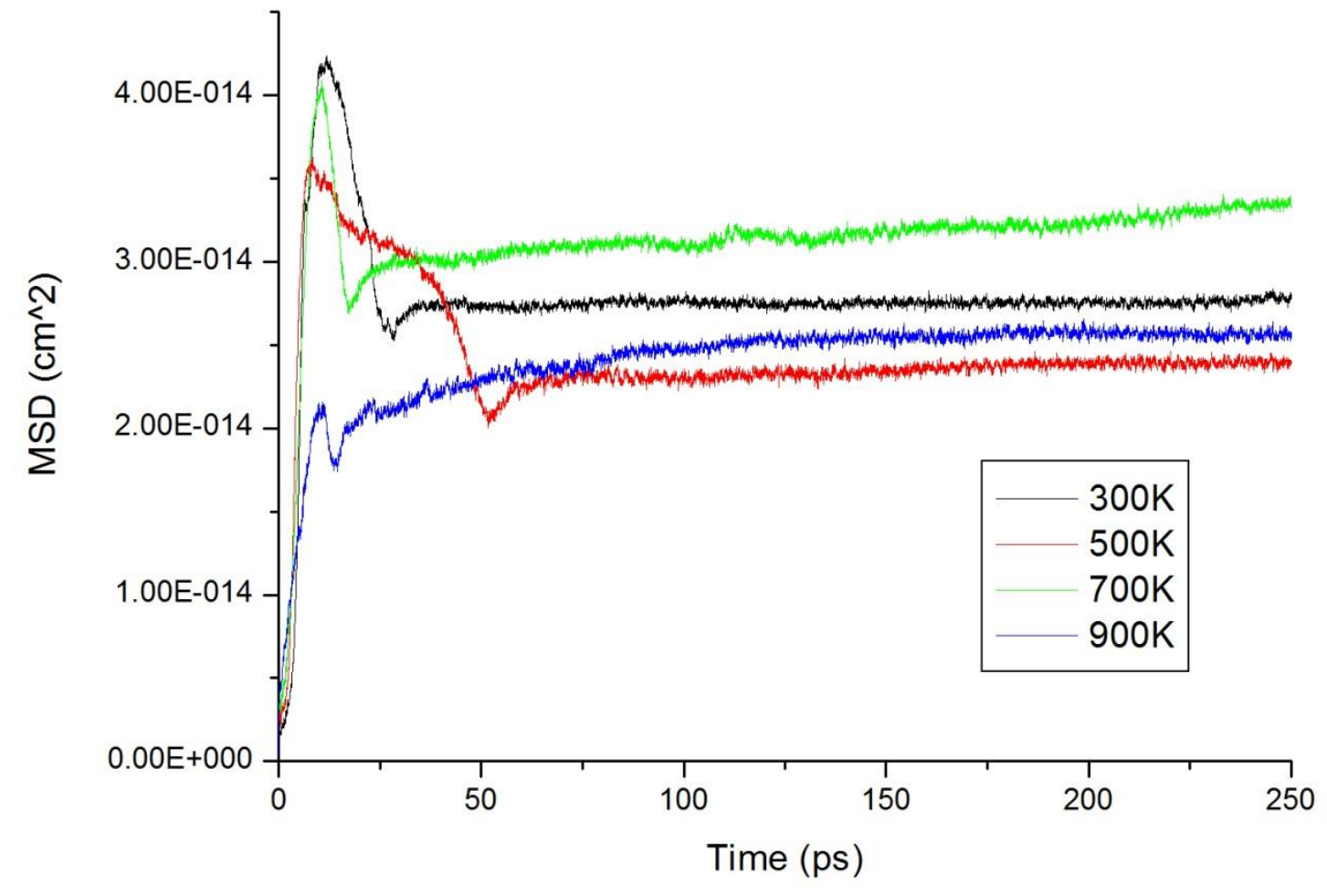


Fig. 5 Porosity of the sintered products with different particle size and temperature. The cases with agglomerations are circled out in the graph.

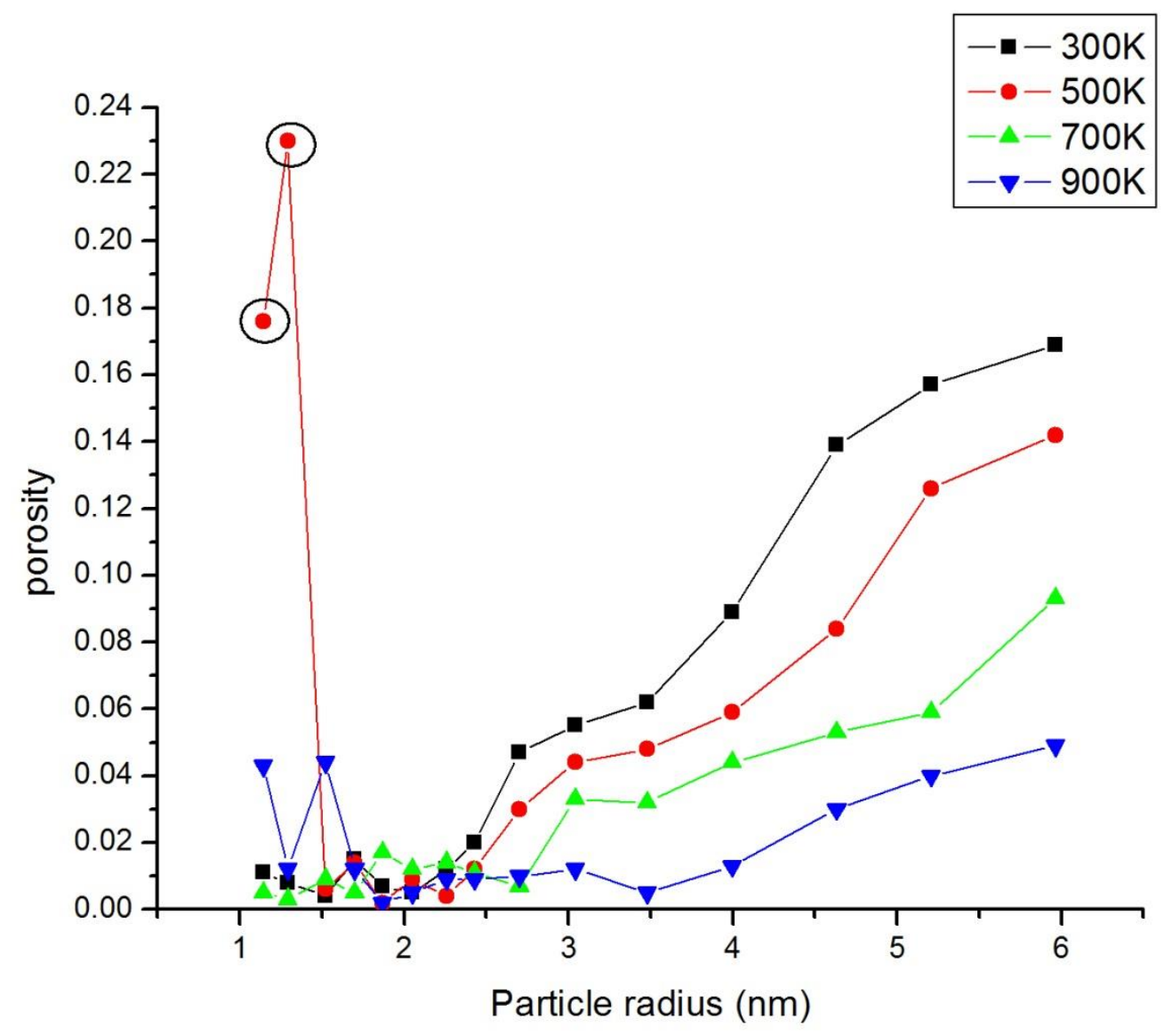


Fig. 6 Porosity of the sintered products under different pressures.

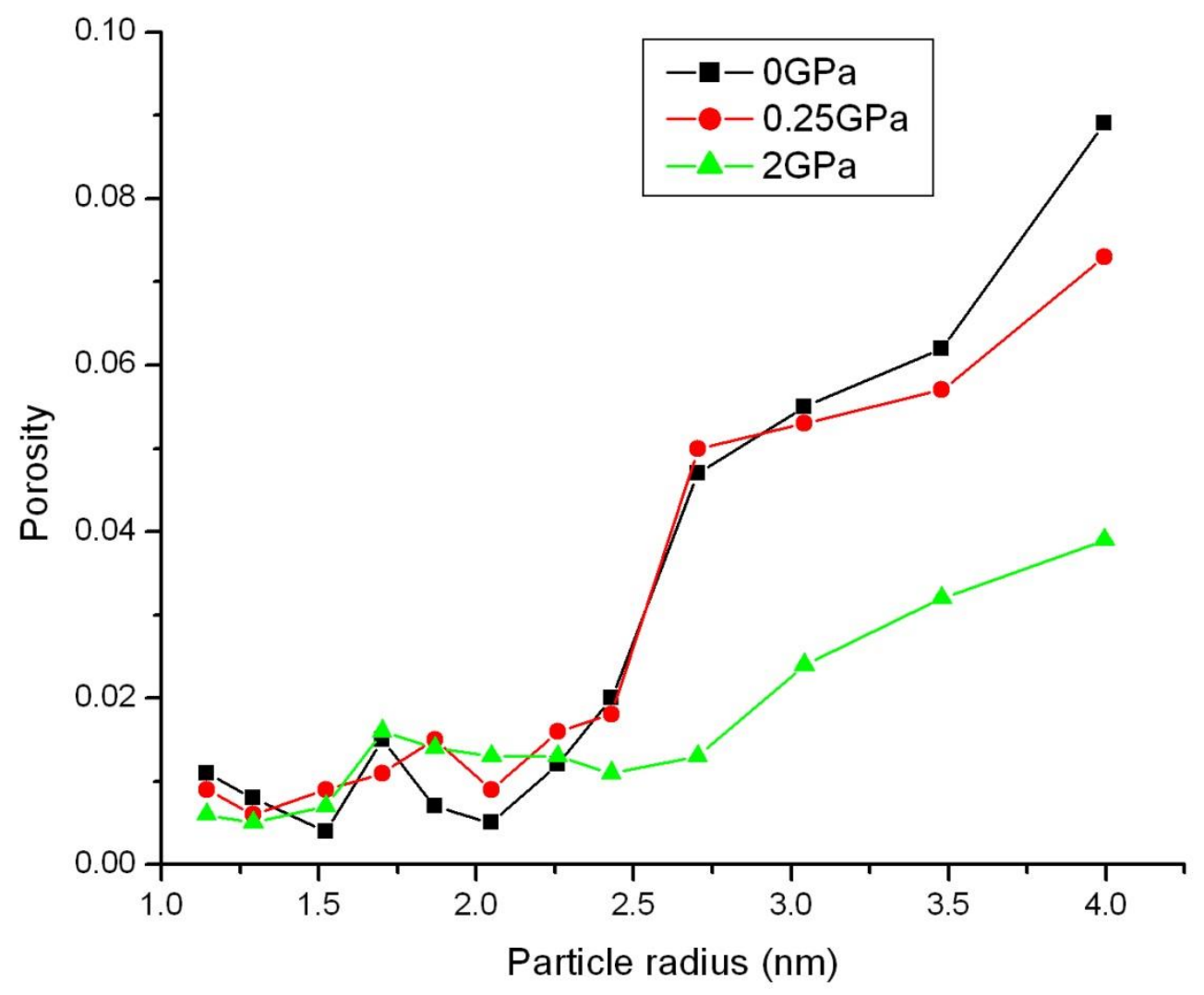


Fig. 7 Porosity of the sintered products with different initial interparticle gaps. The cases with agglomerations are circled out in the graph.

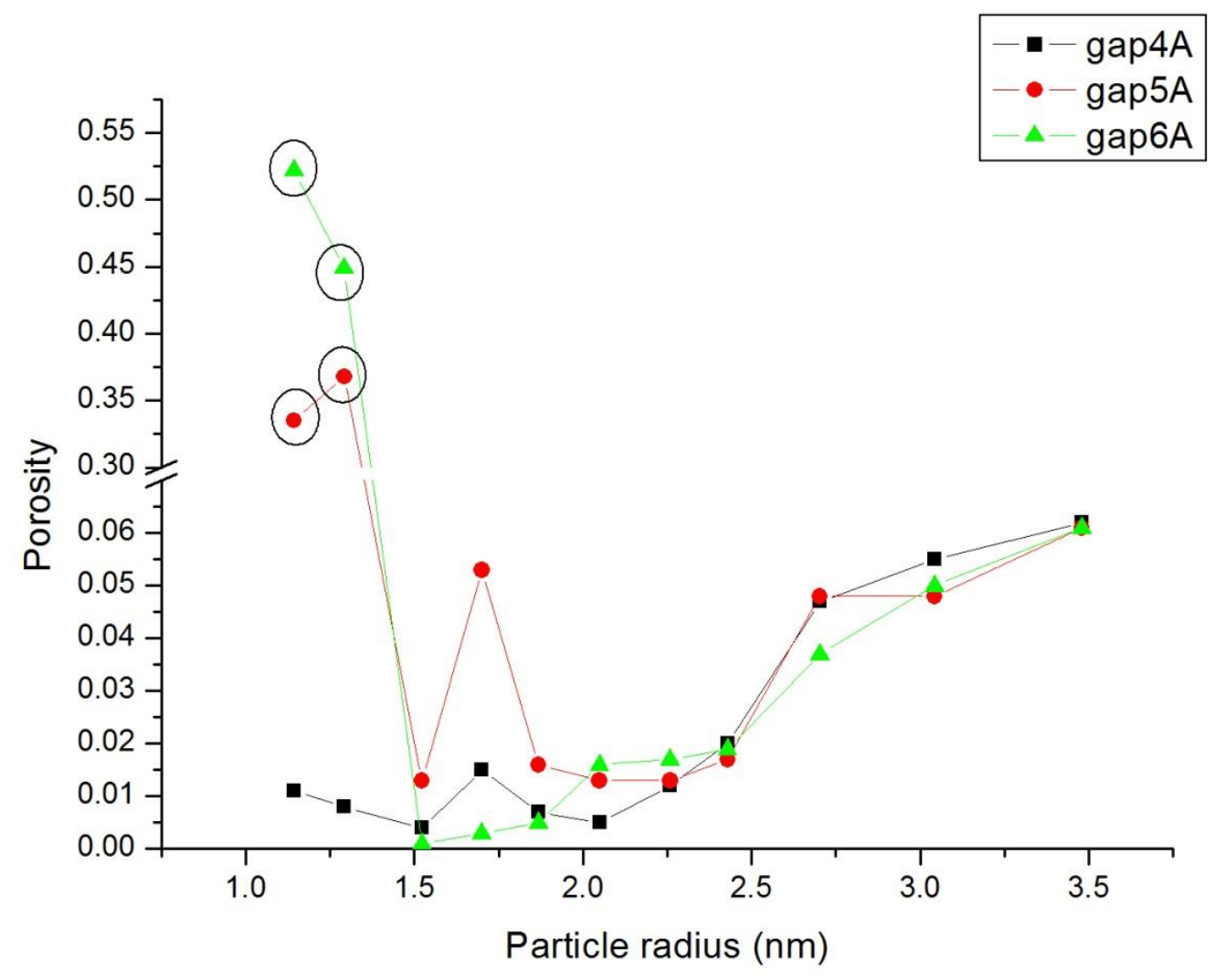


Fig. 8 Grain boundaries in the sintered products. Atoms on grain boundaries and other defects, i.e. those with less on $12 \mathrm{CN}$, are labelled red, and atoms on fcc lattice, i.e. those with $12 \mathrm{CN}$, are labelled blue.

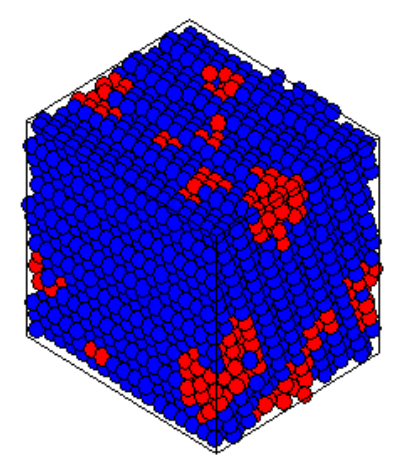

(a)

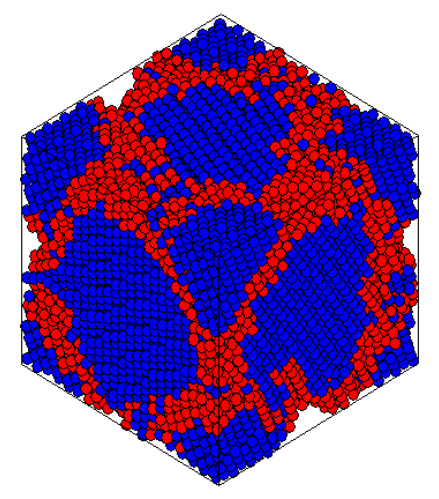

(b)

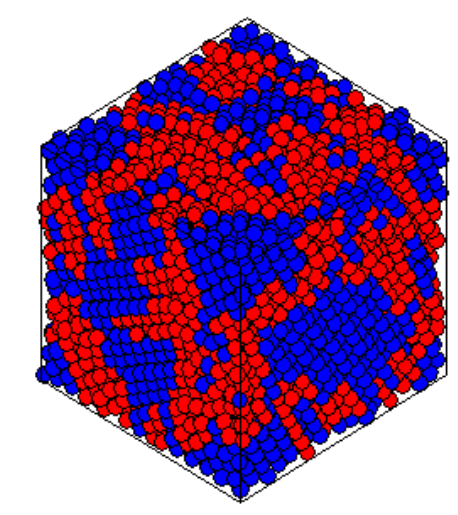

(c) 
Fig. 9 Snapshots of grain boundaries during sintering. Atoms on grain boundaries and other defects, i.e. those with less on $12 \mathrm{CN}$, are labelled red, and atoms on fcc lattice, i.e. those with 12 $\mathrm{CN}$, are labelled blue. Time is indicated below each snapshot.
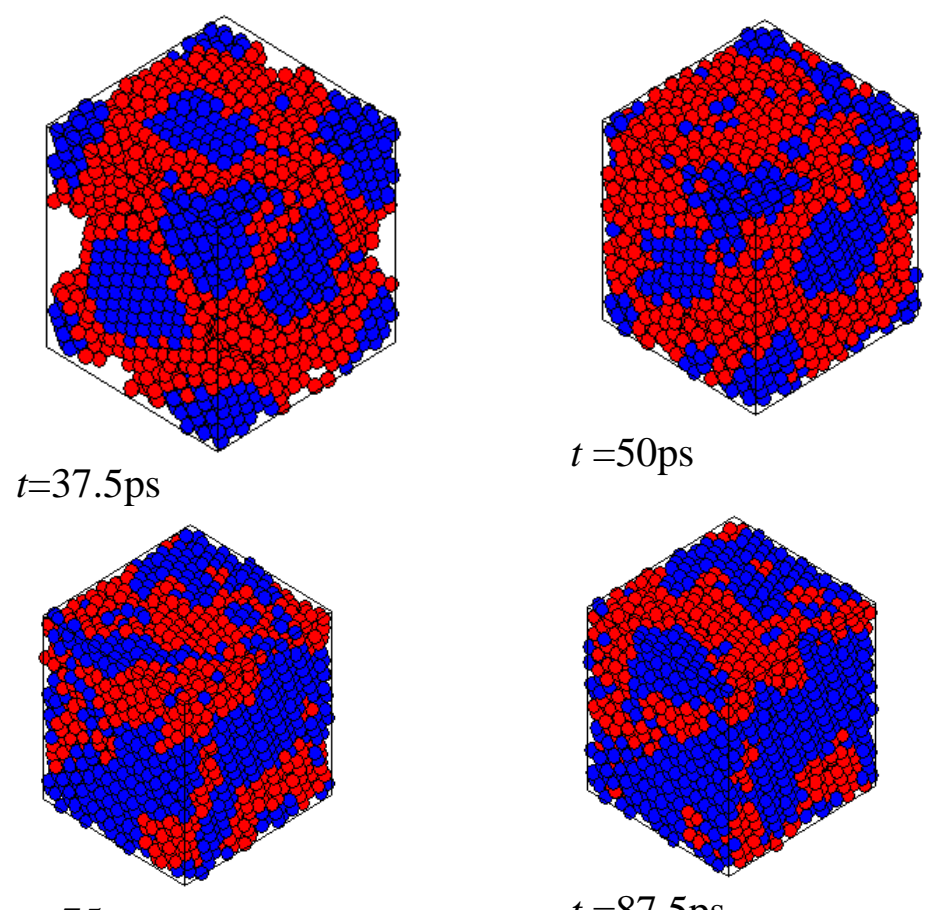

$t=75 \mathrm{ps}$
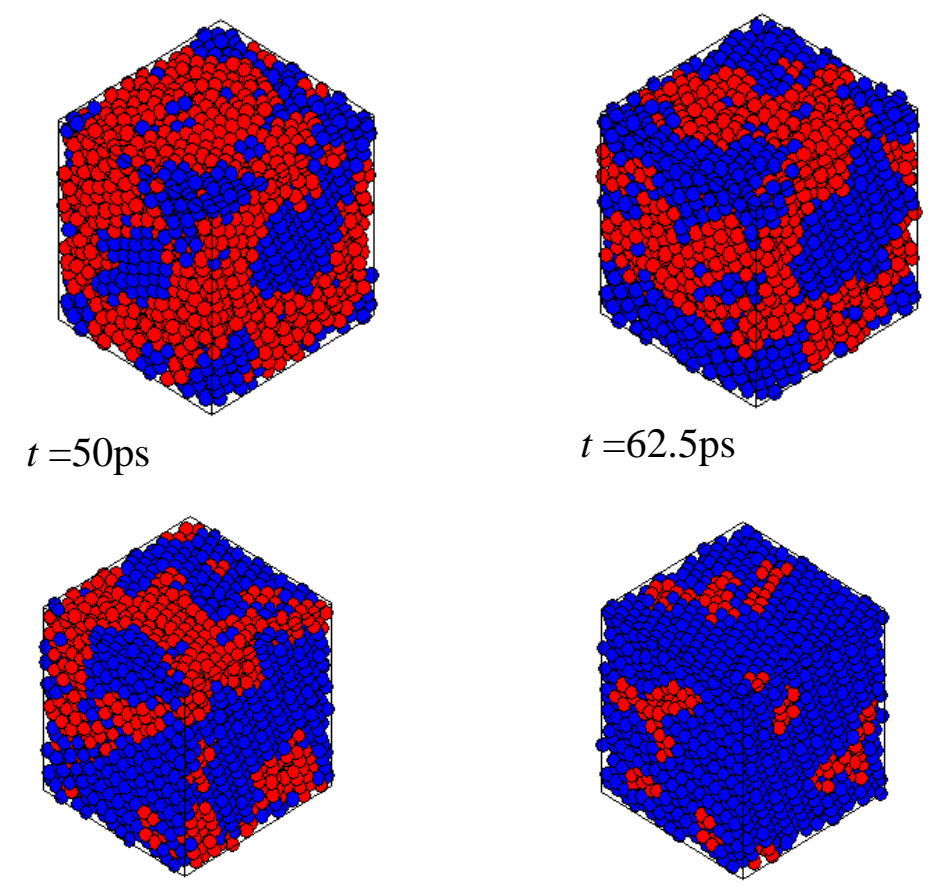

$t=62.5 \mathrm{ps}$

$t=87.5 \mathrm{ps}$

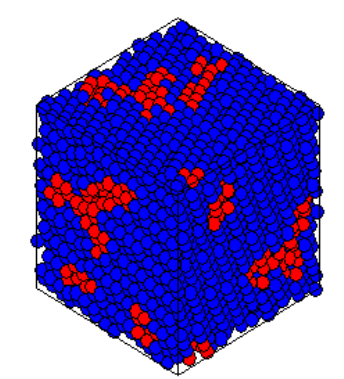

$t=100 \mathrm{ps}$ 
Fig. 10 Shrinkage of two nanoparticles sintered at 300K.

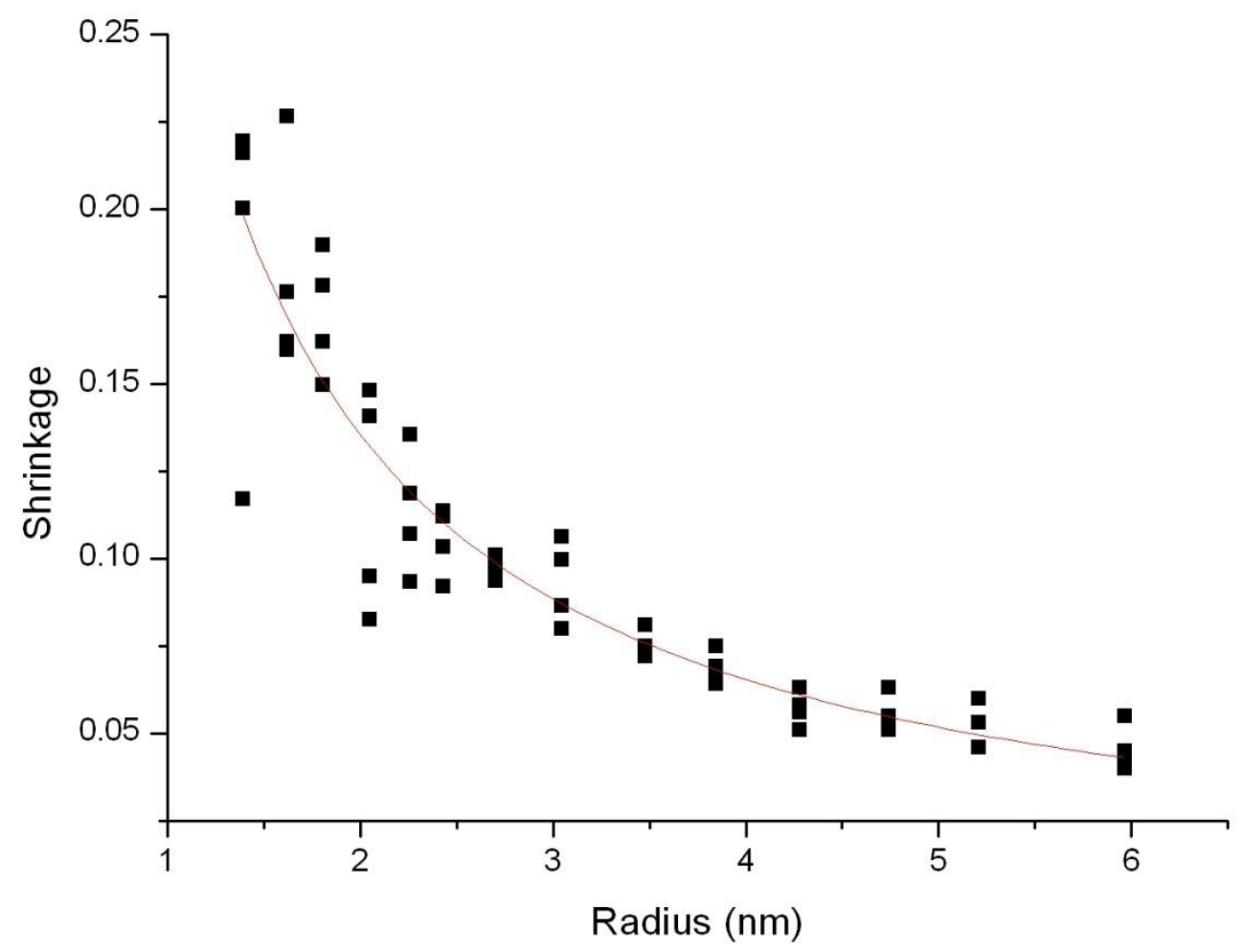


Fig. 11 Porosity derived from two-sphere model and many particle sintering simulations. Red dot: theoretical porosity calculated from two sphere shrinkage. Black line: porosity from many particle sintering simulations. The temperature of the system is $300 \mathrm{~K}$.

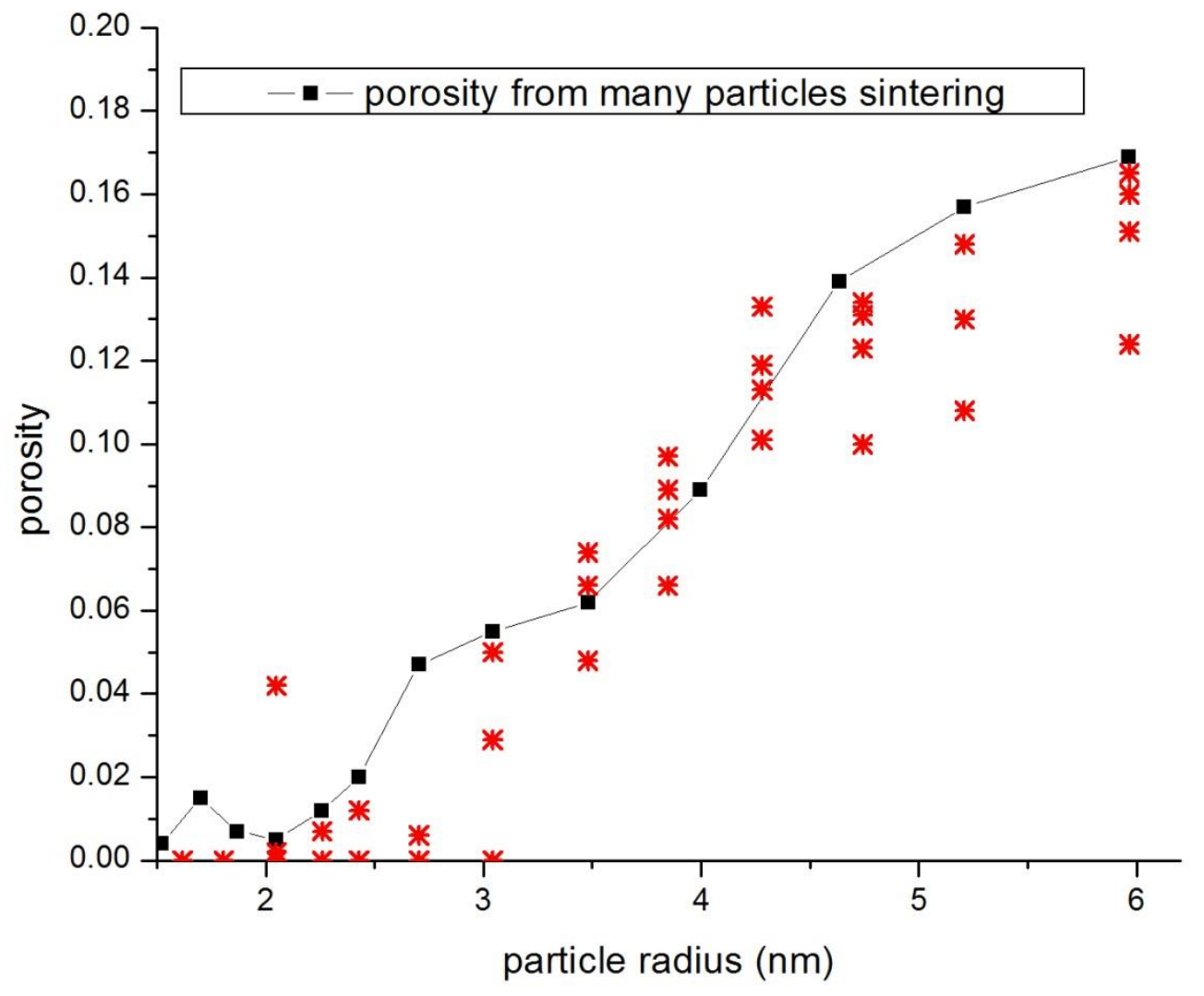


Fig. 12 Shrinkage with respect to time for two $6.0 \mathrm{~nm}$ nanoparticles with same orientation sintered at different temperatures.

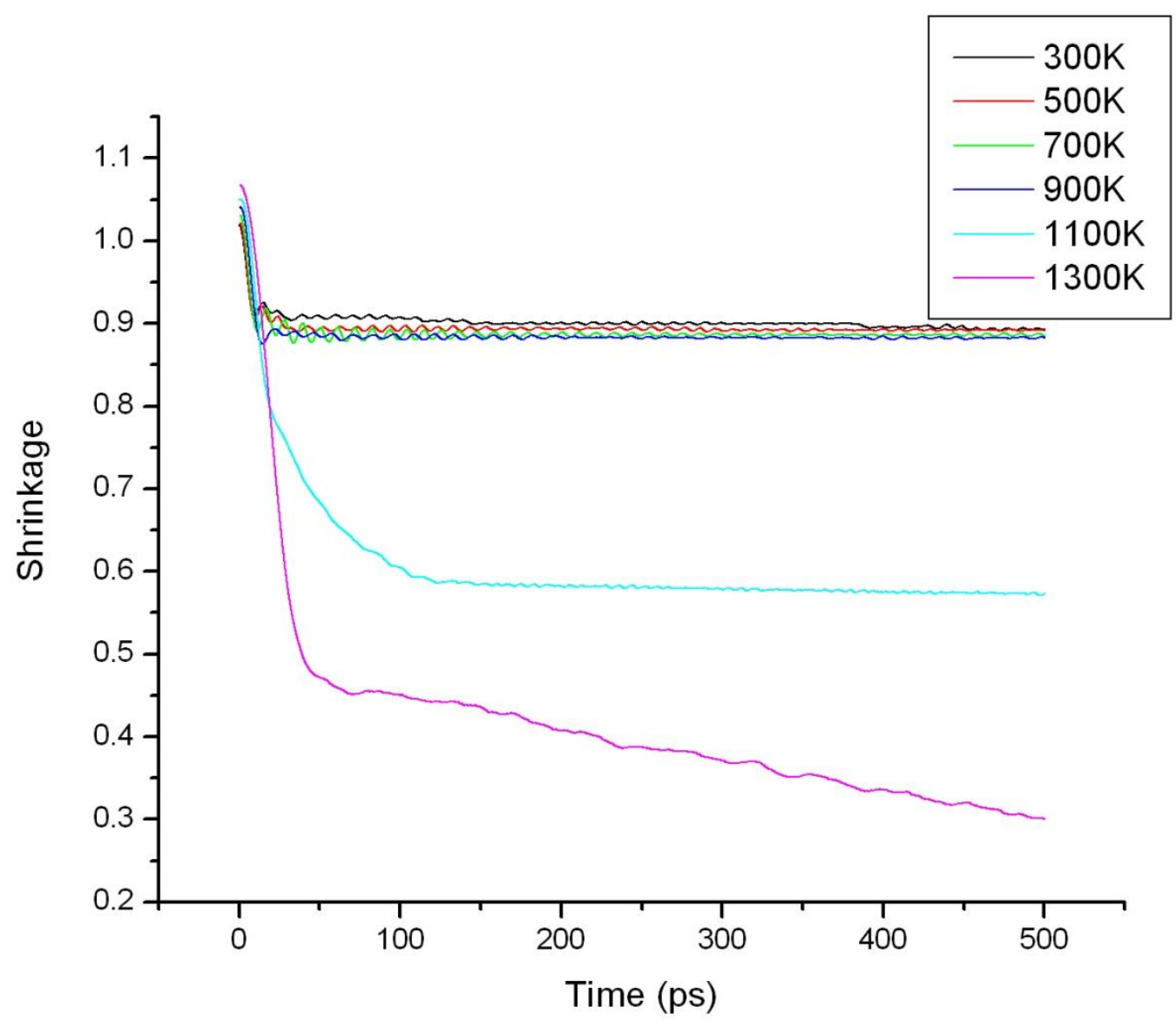


Fig. 13 Average radial distribution of normal stresses at neck during sintering of two nanoparticles each of 10005 atoms at 300K.

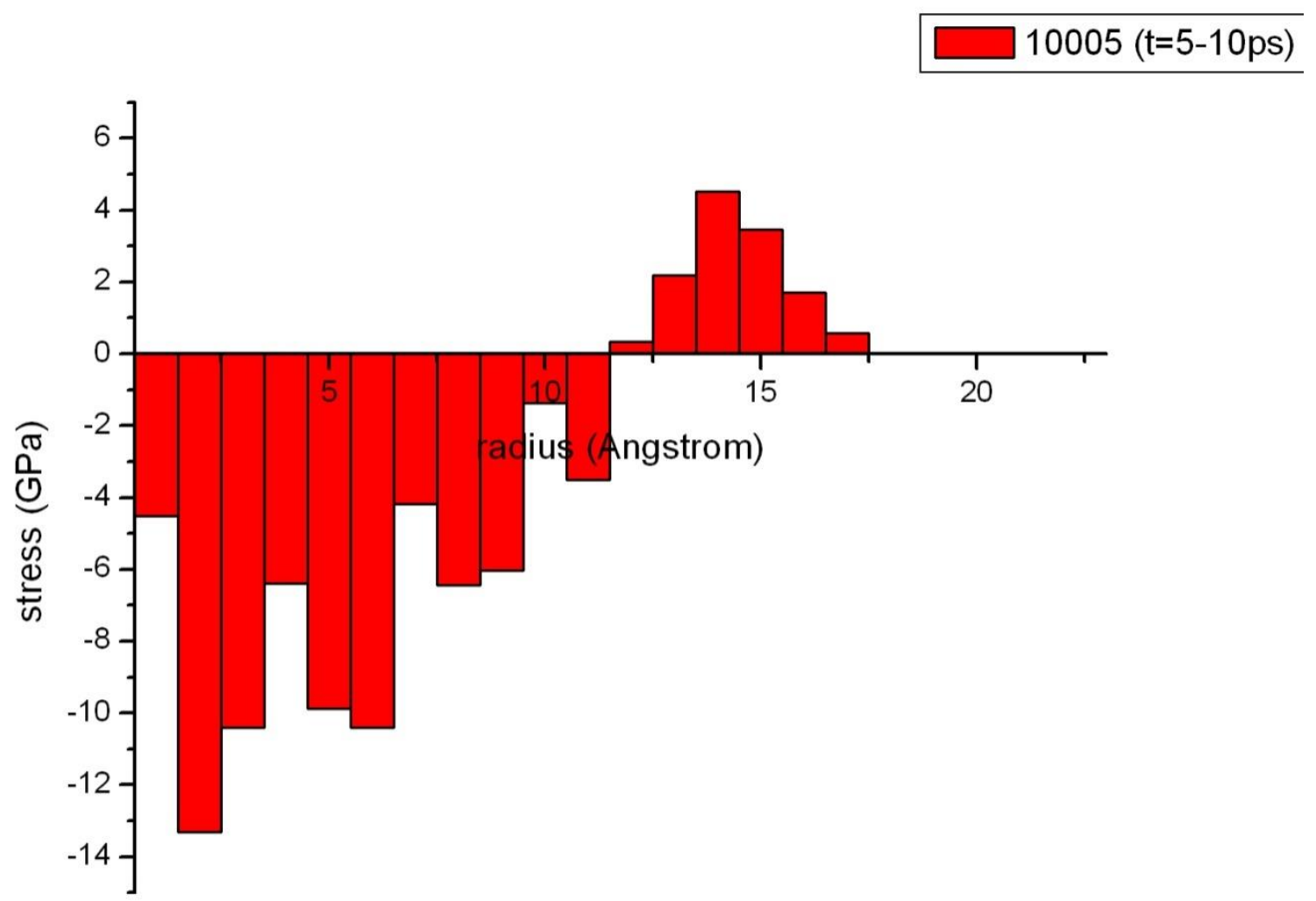


Fig. 14 Relative displacement maps for typical sintered products at the end of the simulation.

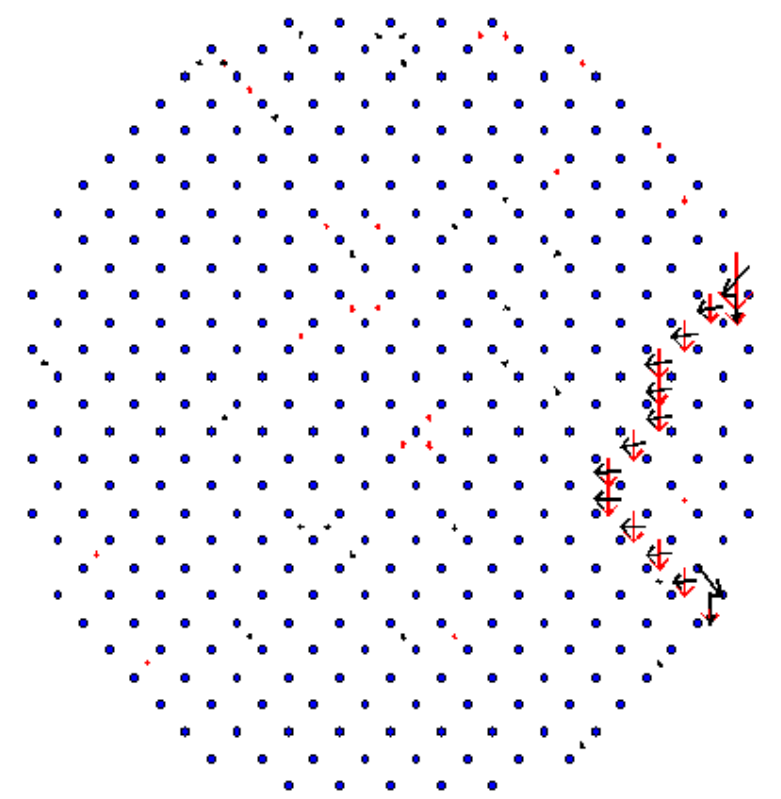

(a) dislocation

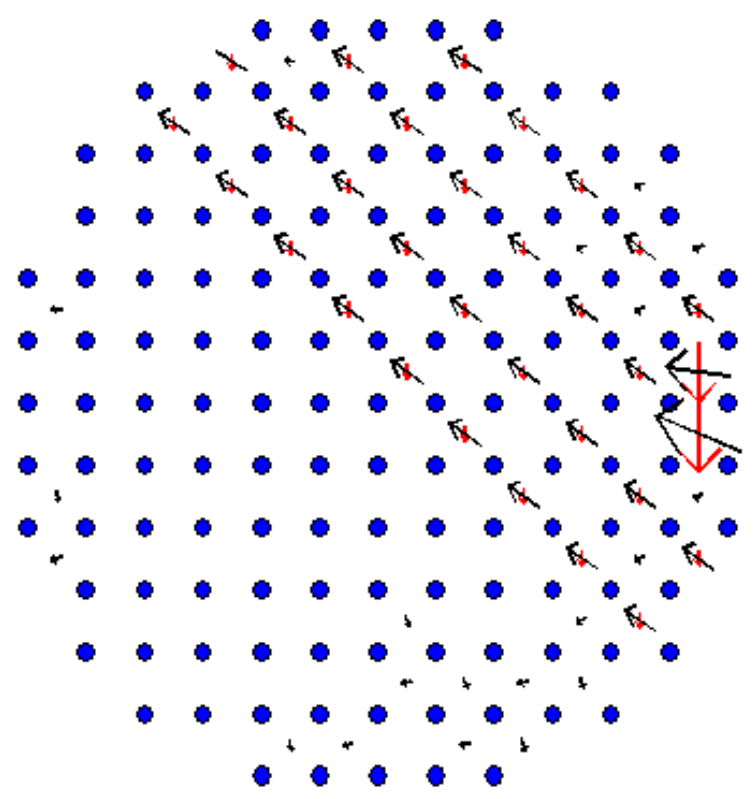

(b) twin 